\title{
Position paper: Impact on medical and health personnel in the SARS-CoV-2 pandemic
}

\section{Documento de postura: la afectación de personal médico y de la salud en la pandemia de SARS-CoV-2}

\author{
Teresita Corona, * Jorge A. Castañón-González, Patricia Clark-Peralta, Carmen García-Peña, \\ Rosalinda Guevara-Guzmán, Guillermo Domínguez-Cherit, José Halabe-Cherem, \\ Malaquías López-Cervantes, Alejandro Macias-Hernández, Mayela Rodríguez-Violante, \\ Patricio Santillán-Doherty, Gilberto Vázquez-de Anda and Alberto Lifshitz Guinzberg. \\ Covid-19 Pandemic Analysis Committee, Academia Nacional de Medicina de Mexico, Ciudad de México, Mexico
}

The National Academy of Medicine of Mexico, as an academic, educational, reflective, representative body of Mexican medicine and as a Federal Government advisory institution, has been advertent to the development of SARS-CoV-2 infection in the country and expresses its interest in contributing to the achievement of the best possible outcomes.

Physicians, owing to the essence of their profession, and like other health workers, are naturally exposed to the risks of transmission posed by contact with infected patients, but an important part of their profession involves learning to protect themselves from these dangers and to avoid disseminating them. Still, as history shows, there are cases where these protective measures are not optimally applied and people end up affected.

The Academy has been sensitive when ascertaining that the COVID-19 pandemic has considerably affected doctors and health personnel, who have experienced, in many countries, including Mexico, a higher-than-expected rate of transmission and deaths, although we do not know the exact figures. More than 130 countries have reported deaths in professionals of this sector; the numbers of infected health workers in international series range from 3.5 to $29 \%$ in China, $10.7 \%$ in Italy and 15.6 to $19 \%$ in the United States. ${ }^{1}$
The impact on medical personnel in this pandemic indicates the existence of an implicit supererogatory duty; i.e., the need to act beyond what the specified obligations demand. Medical professionals have an occupational role that goes beyond duties stipulated in contracts or agreements, as it is governed by ethical principles that go from the historical conception of the doctor-patient relationship itself, passing through professionalism tied to the virtue of honoring commitment to healing, and end with an agreement or consent to participate in a social pact from which powers and obligations that bind them are derived. One of them is the "duty to treat", understood as the provision of care to others despite inherent risks. ${ }^{2}$ The risks assumed by doctors are evident: while the vast majority of the population has been subjected to containment and mitigation policies, medical professionals have not only been holding up at work, but, in many circumstances, they are directly in contact with SARS-CoV-2-infected patients, with exposure to aerosols, areas and supplies contaminated with the virus. This situation has been historically discussed since the beginning of the human immunodeficiency virus epidemic and, more recently, as a result of the severe acute respiratory syndrome, Middle-East respiratory syndrome and Ebola outbreaks; the present pandemic puts us to the test again. 
On the other hand, the deficit of medical personnel in general in Mexico is a well-known fact (where the mean is 2.4 physicians per 1,000 population, when in countries of the Organization for Economic Cooperation and Development it is 3.5$) ;{ }^{3}$ this pandemic has revealed the shortage of personnel capable to manage the excess of serious cases that occur (specialists in intensive care, pulmonology, anesthesiology, infectology, internal medicine, among others).

Supererogation and scarcity support the argument that medical personnel should be considered a valuable resource, the sustainability of which should be looked after to the extreme.

One study conducted at the National Institute of Medical Sciences and Nutrition Salvador Zubirán ${ }^{4}$ shows that health personnel accounts for $13 \%$ of the cases reported in Mexico City, although the number of tests that was carried out was larger in proportion to those performed in the rest of the population. Among the patients treated for this infection at the same Institute, $18.6 \%$ were health workers. ${ }^{5}$ In another study conducted at the National Institute of Geriatrics, ${ }^{6}$ the number of tests performed on health workers is reported to be almost 10 -fold higher than in the general population $(34,349$ vs. 3,825 per 100,000$)$, whereby more asymptomatic and mild cases were identified among health personnel. For that reason, lethality in health personnel was lower than in the general population, although the population mortality rate was higher (195 vs. 125 deaths per 100,000). This constitutes a serious concern for this Academy.

A personnel training policy, with sufficient and adequate access to protective equipment, as well as an occupational medicine program that includes liberal and wide performance of PCR tests for SARS-CoV-2 (in symptomatic personnel and their asymptomatic contacts), can help maintain a nosocomial-origin COVID-19 low prevalence in healthcare personnel.7,8 Part of health workers have acquired the infection in the community (at the National Institute of Respiratory Diseases, 128 out of 3,481 had nosocomial infection, with no deaths, and 231 out of 3,481 acquired it in the community, with two deaths [unpublished data]).

We believe that the measures of care to avoid transmission should not only be applied when doctors and health personnel are exposed to COVID-19-diagnosed patients; in epidemic situations, they should be used when exposed to all types of patients because, among them, there may be some with asymptomatic infection, which is equally transmissible. In addition, training has to be reinforced, preferably through face-to-face activities before health personnel come into contact with people infected with SARS-CoV-2 or with COVID-19, particularly in recently hired staff, and in those who have been specifically reassigned to the care of patients with COVID-19, either in hospitalization or outpatient areas. In general, this personnel, by not being familiarized with the care of critically ill patients with SARS-CoV-2, are vulnerable to infection.

For this reason, the Academy expresses its recommendation to use such measures with all patients and to take to the extreme the recognized care measures, such as that all health personnel assigned to areas designated as COVID have the necessary personal protective equipment at all times: mask, face mask or high-efficiency mask (N95, NK95 or equivalent or superior), as well as eye protection and additional equipment, such as gowns and gloves, as determined by the policies of the institution. All health personnel who have contact with patients, including those who work in areas not designated as COVID, should have access to triple-layer or higher efficacy surgical masks and to additional equipment established by the institution, as well as to local plans and policies in order for work areas to be kept ventilated and to avoid overcrowding.

\section{Measures to prevent health personnel infection}

- All medical personnel should receive specific training in the necessary protection measures according to the activities they are expected to perform.

- Use of three-layer face masks with nose wire all the time.

- Eye protection with safety googles or face shield

- Avoiding that health workers who attend COVID work at different hospitals should be tried, for which improving their remuneration would be advisable.

- Sampling of cases based on suspicious symptoms and contact tracing should be carried out.

- Special monitoring of workers with multiple morbidities and in vulnerable conditions should be continued in order to timely identify the infection and ensure treatment

- The best strategy to protect health workers is by reducing SARS-CoV-2 community circulation. For this, the participation of society is essential, maintaining the measures of face mask universal use and physical distancing, regardless of how much wearing out this implies. 


\section{References}

1. Bandyopadhyay S, Baticulon RE, Kadhum M, Alser M, Ojuka DK, Badereddin $Y$. Infection and mortality of healthcare workers worldwide from COVID-19: a scoping review. medRxiv [Preprint]. 2020 Jun 05. DOI: 10.17605/OSF.IO/JYUXB

2. Daniels N. Just health: meeting health needs fairly. United Kingdom: Cambridge University Health; 2008.

3. Organisation for Economic Co-operation and Development [Internet]. France: Health at a Glance 2019; 2019.

4. Guerrero-Torres L, Caro-Vega Y, Crabtree-Ramírez B, Sierra-Madero JG. Clinical characteristics and mortality of healthcare workers with SARSCoV-2 infection in Mexico City. Clin Infect Dis. 2020.
5. Ortiz-Birzuela E, Villanueva-Reza M, González-Lara MF, Tamez-Torres KM, Román-Montes CM, Díaz-Mejía BA, et al. Clinical and epidemiological characteristics of patients diagnosed with COVID-19 in a tertiary care center in Mexico City: a prospective cohort study. Rev Invest Clin. 2020;72:165-177.

6. Antonio-Villa NE, Bello-Chavolla OY, Vargas-Vázquez A, Fermín-Merínez C, Márquez-Salinas A, Pisanty-Alatorre J, et al. Assessing the burden of COVID-19 amongst healthcare workers in Mexico City: A data-driven call to action. Clin Infect Dis. 2020.

7. Ñamendy-Silva SA. Health care workers on the frontlines of COVID-19 in Mexico. Heart Lung. 2020;29:1.

8. Kaur R, Weiss TT, Perez A, Fink JB, Chen R, Luo F, et al. Practical strategies to reduce nosocomial transmission to healthcare professionals providing respiratory care to patients with COVID-19. Critical Care. 2020;24:571. 